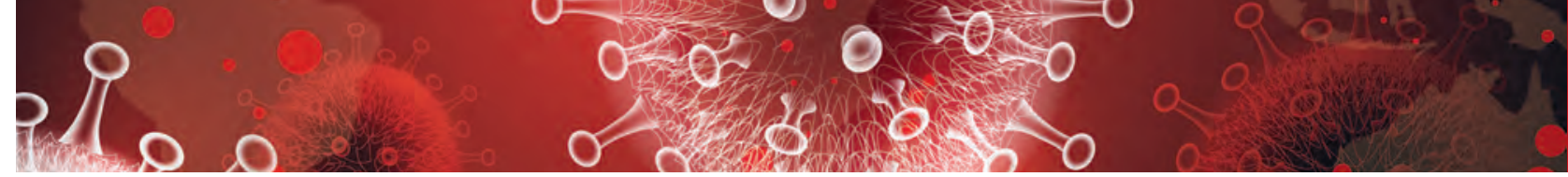

\title{
COVID-19 vaccination during pregnancy: Expert guidance on counseling your patients
}

\author{
Three maternal-fetal medicine specialists weigh in
}

\section{IN THIS ARTICLE}

Dr. Ashley Roman comments

this page

Drs. Ashley Coggins and Jeanne Sheffield comments page 24
E ach clinician has had to develop his or her individual approach and follow their institutional guidance regarding counseling and managing all of their patients' expectations in this quickly changing climate of vaccination availability and recommendations. For pregnant women specifically, who are at increased risk for severe SARS-COV-2 disease, ${ }^{1}$ the availability of varied types of vaccines for COVID-19 is promising, but with limited data available to discuss safety for the mother and the baby, what is the best discussion to guide decision making? OBG MANAGEMENT reached out to several experts, who share here what they do in their practices.

\section{Addressing an uncharted front in the war on COVID-19}

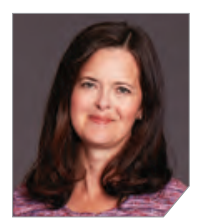

Ashley S. Roman, MD, MPH

Silverman Associate Professor of Obstetrics and Gynecology

Director, Division of Maternal Fetal Medicine

Program Director, Maternal Fetal Medicine Fellowship

Department of Obstetrics and Gynecology

NYU Grossman School of Medicine

NYU Langone Health

New York, New York n December 2020, the US Food and Drug Administration (FDA)'s Emergency Use Authorization of the first COVID-19 vaccine presented us with a new tactic in the war against SARS-COV-2-and a new dilemma for obstetricians. What we had learned about COVID-19 infection in pregnancy by that point was alarming. While the vast majority $(>90 \%)$ of pregnant women who contract COVID-19 recover without requiring hospitalization, pregnant women are at increased

Dr. Roman's comments were published online first May 14, 2021. Drs. Coggins' and Sheffield's comments were published online first May 25, 2021. The authors report no financial relationships relevant to this article.

doi: 10.12788/obgm.0108 risk for severe illness and mechanical ventilation when compared with their nonpregnant counterparts. ${ }^{2}$ Vertical transmission to the fetus is a rare event, but the increased risk of preterm birth, miscarriage, and preeclampsia makes the fetus a second victim in many cases. $^{3}$ Moreover, much is still unknown about the long-term impact of severe illness on maternal and fetal health.

\section{Gaining vaccine approval}

The COVID-19 vaccine, with its high efficacy rates in the nonpregnant adult population, presents an opportunity to reduce maternal morbidity related to this devastating 
FAST

TRACK

The decision
to accept the
coVID-19 vaccine
or not ultimately
belongs to the
patient

The decision

to accept the

COVID-19 vaccine

or not ultimately

patient illness. But unlike other vaccines, such as the flu shot and TDAP, results from prospective studies on COVID-19 vaccination of expectant women are pending. Under the best of circumstances, gaining acceptance of any vaccine during pregnancy faces barriers such as vaccine hesitancy and a general concern from pregnant women about the effect of medical interventions on the fetus. There is no reason to expect that either the mRNA vaccines or the replication-incompetent adenovirus recombinant vector vaccine could cause harm to the developing fetus, but the fact that currently available COVID-19 vaccines use newer technologies complicates the decision for many women.

Nevertheless, what we do know now is much more than we did in December, particularly when it comes to the mRNA vaccines. To date, observational studies of women who received the mRNA vaccine in pregnancy have shown no increased risk of adverse maternal, fetal, or obstetric outcomes. ${ }^{4}$ Emerging data also indicate that antibodies to the SARS-CoV-2 spike protein-the target of all 3 vaccines-is present in cord blood, potentially protecting the infant in the first months of life from contracting COVID-19 if the mother receives the vaccine during pregnancy ${ }^{5,6}$

\section{Our approach to counseling}

How can we best help our patients navigate the risks and benefits of the COVID-19 vaccine? First, by acknowledging the obvious: We are in the midst of a pandemic with high rates of community spread, which makes COVID-19 different from any other vaccine-preventable disease at this time. Providing patients with a structure for making an educated decision is essential, taking into account (1) what we know about COVID-19 infection during pregnancy, (2) what we know about vaccine efficacy and safety to date, and (3) individual factors such as: - the presence of comorbidities such as obesity, heart disease, respiratory disease, and diabetes

- potential exposures-"Do you have children in school or daycare? Do childcare providers or other workers come to your home? What is your occupation?"

- the ability to take precautions (social distancing, wearing a mask, etc).

All things considered, the decision to accept the COVID-19 vaccine or not ultimately belongs to the patient. Given disease prevalence and the latest information on vaccine safety in pregnancy, I have been advising my patients in the second trimester or beyond to receive the vaccine with the caveat that delaying the vaccine until the postpartum period is a completely valid alternative. The most important gift we can offer our patients is to arm them with the necessary information so that they can make the choice best for them and their family as we continue to fight this war on COVID-19.

\section{Benefits outweigh the risks, for now}

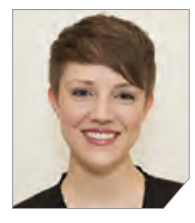

Ashley S. Coggins, MD

Fellow, Division of Maternal-Fetal

Medicine

Department of Gynecology and

Obstetrics

Johns Hopkins Medicine

Baltimore, Maryland

V

accines have been a lifesaving public health measure since $1000 \mathrm{CE}$, when the Chinese first used smallpox inoculations to induce immunity. ${ }^{?}$

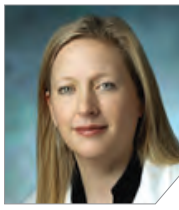

Jeanne S. Sheffield, MD

Professor, Gynecology and Obstetrics

Director, Division of Maternal-Fetal Medicine

Department of Gynecology and Obstetrics

Johns Hopkins Medicine

Work by pioneers such as Edward Jenner, Louis Pasteur, and Maurice Hilleman has averted countless millions of vaccinepreventable illnesses and deaths, and 
FAST

TRACK

Strongly consider all available

COVID-19

vaccination

formulations for

pregnant women

with comorbidities

that place them

at higher risk for

severe COVID-19

infection vaccines have become a routine part of health maintenance throughout the human life cycle.

Pregnant patients who receive vaccines often have an added benefit of protection provided to their infants through passive transfer of antibodies. Several vaccine platforms have been utilized in pregnancy with well-documented improvements in maternal and obstetric outcomes as well as improved neonatal outcomes in the first several months of life.

\section{Risks of COVID-19 in pregnancy}

The COVID-19 pandemic placed a spotlight on medically at-risk groups. Pregnant women are 3 times more likely to require admission to the intensive care unit, have increased requirement for extracorporeal membrane oxygenation treatment, and are up to $70 \%$ more likely to die than nonpregnant peersand this risk increases with the presence of additional comorbidities.

In the case of COVID-19, vaccination trials that have shaped worldwide clinical practice unfortunately followed the historical trend of excluding pregnant patients from participation. This has required clinicians to guide their patients through the decision of whether or not to accept vaccination without having the same reassurances regarding safety and effectiveness afforded to their nonpregnant counterparts. With more than 86,000 pregnant women infected with COVID-19 through April 19, 2021, this lack of information regarding vaccine safety in pregnancy is a significant public health gap. ${ }^{8}$

\section{COVID-19 vaccines}

The current COVID-19 vaccines approved for use in the United States under an Emergency Use Authorization issued by the FDA are nonreplicating and thus cannot cause infection in the mother or fetus. These are

\section{References}

1. Zambrano LD, Ellington S, Strid S, et al. Update: characteristics of symptomatic women of reproductive age with laboratory-confirmed SARS-CoV-2 infection by pregnancy status-United States, January 22-October 3, 2020. 2020;69:1641-1647.

2. Allotey J, Stallings E, Bonet M, et al. Clinical manifestations, risk factors and maternal and perinatal outcomes of coronavirus the Pfizer-BioNTech mRNA vaccine, the Moderna mRNA-1273 vaccine, and the Janssen Biotech Inc. monovalent vaccine. Furthermore, in animal studies that included the Pfizer-BioNTech, Moderna, or Janssen COVID-19 vaccines, no fetal, embryonal, female reproductive, or postnatal development safety concerns were demonstrated.

As of April 19, 2021, 94,335 pregnant women had received a COVID-19 vaccination, and 4,622 of these enrolled in the Centers for Disease Control and Prevention (CDC)'s V-safe Vaccine Pregnancy Registry. ${ }^{9}$ The data reported noted no unexpected pregnancy or infant outcomes related to COVID-19 vaccination in pregnancy. Adverse effects of the vaccine were similar to those in nonpregnant cohorts. Additionally, emerging data suggest passage of immunity to neonates, with maternal antibodies demonstrated in cord blood at time of delivery as well as in breast milk. ${ }^{10}$ To date, these data mainly have come from women immunized with the Moderna and Pfizer-BioNTech mRNA vaccines.

\section{Counseling pregnant patients}

Our counseling aligns with that of the American College of Obstetricians and Gynecologists, the Society for Maternal-Fetal Medicine, and the CDC's Advisory Committee on Immunization Practices. These organizations advise that COVID-19 vaccination should not be withheld from pregnant patients or patients who want to become pregnant. In pregnant patients with comorbidities that place them at higher risk for severe COVID19 infection, all available formulations of the COVID-19 vaccination should be strongly considered. As evidence for vaccination safety continues to emerge, patients should continue to discuss their individual needs for vaccination in a shared decision-making format with their obstetric providers.

\footnotetext{
disease 2019 in pregnancy: living systematic review and metaanalysis. BMJ. 2020;370:m3320. doi: 10.1136/bmj.m3320.

3. Soheili M, Moradi G, Baradaran HR, et al. Clinical manifestation and maternal complications and neonatal outcomes in pregnant women with COVID-19: a comprehensive evidence synthesis and meta-analysis. J Matern Fetal Neonatal Med. February 18, 2021. doi: 10.1080/14767058.2021.1888923.
} 


\section{COVID-19 vaccination during pregnancy}

4. Shimabukuro TT, Kim SY, Myers TR, et al. Preliminary findings of mRNA Covid-19 vaccine safety in pregnant persons. New EnglJ Med. April 21, 2021. doi: 10.1056/NEJMoa2104983.

5. Mithal LB, Otero S, Shanes ED, et al. Cord blood antibodie following maternal COVID-19 vaccination during pregnancy. Am J Obstet Gynecol. 2021;S0002-9378(21)00215-5. doi: 10.1016/j.ajog.2021.03.035.

6. Rottenstreich A, Zarbiv G, Oiknine-Djian E, et al. Efficient maternofetal transplacental transfer of anti- SARS-CoV-2 spike antibodies after antenatal SARS-CoV-2 BNT162b2 mRNA vaccination. Clin Infect Dis. 2021;ciab266. doi: $10.1093 / \mathrm{cid} / \mathrm{ciab} 266$

7. Boylston A. The origins of inoculation. $J \quad R$ Soc Med.
2012;105:309-313.

8. Centers for Disease Control and Prevention. COVID data tracker. Data on COVID-19 during pregnancy: severity of maternal illness. https://covid.cdc.gov/covid-datatracker/\#pregnant-population. Accessed April 19, 2021.

9. Centers for Disease Control and Prevention. V-safe COVID19 Vaccine Pregnancy Registry. https://www.cdc.gov /coronavirus/2019-ncov/vaccines/safety/vsafepregnancy registry.html. Updated May 3, 2021. Accessed April 19, 2021.

10. Gray KJ, Bordt EA, Atyeo C, et al. COVID-19 vaccine response in pregnant and lactating women: a cohort study. Am Obstet Gynecol. 2021;S0002-9378(21)00187-3. doi: 10.1016/j. ajog.2021.03.023. 